\title{
Stokes Drift: A New Estimate
}

\section{Kern E. Kenyon}

4632 North Lane, Del Mar, CA, USA

Correspondence to: Kern E. Kenyon, kernken@aol.com

Keywords: Stokes Drift, New Value

Received: November 19, 2018 Accepted: December 11, 2018

Published: December 14, 2018

Copyright (C) 2018 by author and Scientific Research Publishing Inc.

This work is licensed under the Creative Commons Attribution International License (CC BY 4.0).

http://creativecommons.org/licenses/by/4.0/

\section{Open Access}

\section{ABSTRACT}

A new estimate of the Stokes drift is made based on a model that earlier predicted the pressure fluctuations of surface gravity waves to die away with increasing depth significantly faster than the velocity fluctuations do. At the surface, the Stokes drift is calculated to be very nearly twice the classical value of 1847 . Measurements will be needed to decide which value comes closer to the truth.

\section{INTRODUCTION}

Observations of propagating surface gravity waves show that all their characteristic features diminish monotonically with increasing distance down from the air/water interface. For depths exceeding about one wavelength, instruments that measure pressure or velocity can no longer detect the movement on the surface. Awareness of these facts has been happening over several hundred years.

The orbital motion of the fluid particles also decreases with increasing depth. It is due to this circumstance that the circular orbits are not quite closed: a slow movement of fluid mass takes place in the direction of wave propagation. This small forward motion has come to be called the Stokes drift, probably because Stokes obtained a mathematical expression for it in 1847 [1]. Connecting the Stokes drift with linear momentum, and the concept that these waves transport linear momentum as well as energy, came along much later [2]. Because of the orbital particle motion, the waves transport angular momentum also, which in the past has not always been considered self-evident.

What the exact form the decrease with increase of depth takes for the wave properties does not matter. A Stokes drift will occur if the particle motion is orbital and the rate of decrease is exponential, as Stokes used, or some other way as explored in [3].

To come up with the drift quantitatively, Stokes used a mathematical method and arrived at his algebraic formula. Or it could have been an accidental outcome of the mathematics; the original paper does not make that point clear. Unfortunately, the method itself is a bit complicated. It occurs to me to try a different approach, given below, that is more physical and shorter. After that is done, the two independent results are compared and discussed. No perturbation expansions are employed here and the usual irrotational assumption is not made. 


\section{DEVELOPMENT}

The first three steps (equations) of the development are taken from Section 2 of Reference [3], but then a branching away occurs toward the Stokes drift.

Adopt the reference frame in which the wave shape is fixed and the water flows under the wave surface past the observer. Bernoulli's equation for steady frictionless flow along a streamline is

$$
p=\text { const }-\frac{1}{2} \rho V^{2}-\rho g z
$$

where $p$ is the pressure, $V$ is the speed of flow, $\rho$ is the constant fluid density and $g$ is the acceleration of gravity. At the top of the crest the $z$ axis points up.

The cross-stream force balance at the top of the crest is

$$
\frac{\mathrm{d} p}{\mathrm{~d} z}+\rho g=\frac{\rho V^{2}}{R}
$$

where $R$ is the radius of curvature of the streamlines and it is a function of $z$.

Between (1) and (2) the pressure can be eliminated by first taking the $z$ derivative of (1) and then combining that with (2), which produces

$$
\frac{\mathrm{d} V}{\mathrm{~d} z}=-\frac{V}{R}
$$

Both sides of which are functions of $z$. From (3) new manipulations follow.

When both sides of (3) are multiplied by $2 a$, twice the wave amplitude, the left side is essentially the Stokes drift, because it is then the vertical difference in horizontal velocity over the diameter of particle orbits at the surface. That is so since the forward motion at the crest slightly exceeds the backward motion in the trough by just this amount, in the reference frame attached to the bottom of the ocean, for example.

Therefore, the Stokes drift $U$ at the surface is

$$
U=2 a \frac{\mathrm{d} V}{\mathrm{~d} z}=\frac{2 a V}{R}
$$

Insert into $R$ on the RHS of (4), where the minus sign has been dropped for convenience, the radius of curvature at the surface of a sinusoidal wave of wave number $k\left(R=\frac{1}{a k^{2}}\right)$, without approximation, to get

$$
U=2(a k)^{2} V
$$

Compare (5) with the classical expression $U^{\prime}$ from Stokes

$$
U^{\prime}=(a k)^{2} c
$$

where $c$ is the wave phase speed. For small mean wave slopes $(a k \ll 1) V$ is smaller than $c$ at the crest but not very much smaller, certainly not by a factor of $1 / 2$. Consequently it has been found that the new estimate (5) is almost double the classical result!

\section{DISCUSSION}

Since the Stokes drift is a relatively small quantity, there are difficulties measuring it. In any case a laboratory situation would be needed. However, a factor of two difference between two small results might turn out to be possible to distinguish in a wave tank. Work for the future possibly.

For the present purposes the depth decay rate of the Stokes drift itself, which Stokes found to be proportional to $\mathrm{e}^{2 k z}$, is not needed, but nevertheless it is forecast from the analysis in [3] that the depth decay associated with (5) will not in all probability have an exponential rate. Therefore the Stokes transport, involving a vertical integration of the Stokes drift, very likely will not turn out to agree with the classical result. 
There has been academic interest in the Stokes drift for a considerable period of time, but practical applications exist as well. For example, plastic trash and other flotsam landing on beaches are brought there partly by surface gravity waves which get it to the point where breaking waves bring it the rest of the way. At most beaches around the world waves are observed to come in to shore but rarely does a person see a wave go back out to sea.

\section{CONCLUSION}

By a short derivation, using more physics than mathematics, a value for the Stokes drift of a progressive surface gravity wave is obtained which is about two times as large as the one that has been the standard for over 170 years. Future data from wave tank studies, presumably, may be able to decide which value comes closer to reality.

\section{ACKNOWLEDGEMENTS}

Without initial encouragement from Wilton Sturges, followed by helpful comments on the text, this manuscript probably would not have been composed.

\section{CONFLICTS OF INTEREST}

The author declares no conflicts of interest regarding the publication of this paper.

\section{REFERENCES}

1. Stokes, G.G. (1847) On the Theory of Oscillatory Waves. Transactions of the Cambridge Philosophical Society, 8, 441-455.

2. Barnett, T.P. and Kenyon, K.E. (1975) Recent Advances in the Study of Wind Waves. Reports on Progress in Physics, 38, 667-729. https://doi.org/10.1088/0034-4885/38/6/001

3. Kenyon, K.E. (2013) Depth Decay Rate for Surface Gravity Wave Pressure and Velocity. Natural Science, 5, 44-46. https://doi.org/10.4236/ns.2013.51007 